\title{
Insulinoma presenting as refractory seizure disorder
}

\author{
S Pathmanthan ${ }^{1}$, D U S Bulugahapitiya ${ }^{2}$, A S K Banagala ${ }^{3}$, H S K Ruwanpathirana ${ }^{4}$, G P Karunasekara ${ }^{5}$
}

Sri Lanka Journal of Diabetes, Endocrinology and Metabolism 2012; 1: 46-48

\begin{abstract}
Diagnosis of insulinoma may be delayed when symptoms are nonspecific. Rarely neuroglycopenic symptoms are the primary feature and these patients can be misdiagnosed as having epilepsy or neuropsychiatric disease. We report a case of insulinoma presenting as an adult-onset refractory seizure disorder. The time from onset of symptoms to diagnosis was 9 years. The atypical features of the episodes of hypoglycaemia, and the poor response to treatment led to a review of diagnosis. This case highlights the importance of considering hypoglycaemia in atypical neurological or psychiatric presentations.
\end{abstract}

\section{Introduction}

Insulinoma is a rare neuroendocrine tumour arising from beta cells of islets of langerhans with an overall incidence of 4 cases per million per year. About $90 \%$ of insulinomas are solitary, benign, intrapancreatic and sporadic while up to $10 \%$ could be multiple, malignant, extrapancreatic and familial. The familial insulinomas have a special predilection for occurrence in association with MEN - 1 and von Hippel - Lindau disease (1-3).

Clinical diagnosis of hypoglycaemia is confirmed with biochemical investigations. Imaging methods are useful in localisation of the insulinoma. However, localisation can be difficult as the symptoms usually precede occurrence of a tumour that can be visualised on imaging studies $(4,5)$. Surgical excision is the treatment of choice and is curative in most cases $(6,7,8)$.

\section{Case report}

A 32-year old female presented with frequent episodes of loss of consciousness and generalised tonic clonic fits of 9 years duration. The seizures were preceded by sweating, giddiness, and palpitations. A diagnosis of epilepsy had been made 9 years ago and despite gradual up titration and treatment with 3 anti epileptic drugs there was no resolution of the seizures. Her appetite increased over the past few years and her weight increased by 20 Kg. She also had secondary amenorrhoea since 1999 but denied any galactorrhoea or visual changes. There was no family history of hyperparathyroidism, ulcer disease, or hypoglycaemia. One sibling had diabetes mellitus. Physical examination revealed obesity (BMI 36.96), and acanthosis nigricans, but no other abnormalities.

Serum glucose level observed during an episode was $1.1 \mathrm{mmol} / \mathrm{L}$. Administration of intravenous glucose resulted in relief of symptoms. When plasma glucose level was 1.1 $\mathrm{mmol} / \mathrm{L}$, serum insulin level was $73.80 \mu \mathrm{IU} / \mathrm{ml}$ (normal range: $<28.4 \mu \mathrm{IU} / \mathrm{ml}$ ) and serum C-peptide level was 5.6 $\mathrm{ng} / \mathrm{ml}$ (normal range: $0.9-7.1 \mathrm{ng} / \mathrm{ml}$ ). Her pituitary adrenal axis was intact. Plasma thyroid-stimulating hormone (TSH), prolactin, follicle stimulating hormone (FSH) and leuteinizing hormone $(\mathrm{LH})$ levels were within normal range. In view of the clinical features and results of laboratory investigations, diagnosis of an autonomous insulin secreting tumour was made.

Abdominal ultrasound (US) did not reveal any pancreatic lesion. A triple phase computed tomography (CT) of the abdomen demonstrated a well-defined $2.2 \times$ $2.4 \times 3.0 \mathrm{~cm}$ soft-tissue density mass in the head of the pancreas. The lesion was isodense on plain scan and showed early enhancement in arterial phase and appeared isodense to pancreas in venous phase of the contrast study. Scattered microcalcification within the tumour was noted too.

Whipple's pancreatico duodendectomy was performed due primarily to the relatively large size of this tumour and the presence of microcalcification within the tumour. Intra-operative ultrasound scan was performed during surgery to confirm the location of the tumour and to exclude presence of multiple tumours. Encapsulated

${ }^{1}$ Senior Registrar in Endocrinology, National Hospital of Sri Lanka, Colombo, ${ }^{2}$ Consultant Endocrinologist, Colombo South Teaching Hospital, ${ }^{3}$ Consultant Surgeon, Colombo South Teaching Hospital, ${ }^{4}$ Consultant Physician, Colombo South Teaching Hospital, ${ }^{5}$ Consultant Dermatologist, Colombo South Teaching Hospital. 
tumour was found within the pancreatic tissue, attached to the outer surface of the duodenal wall. Immediately after removal of the mass, serum glucose level increased to 10.8 $\mathrm{mmmol} / \mathrm{L}$. Hyperglycaemia was observed during the first 24 hours and intravenous insulin infusion commenced. Normoglycaemic status was achieved with human insulin. Insulin was omitted on post-op day 15 and the patient experienced no further hypoglycaemic episodes.

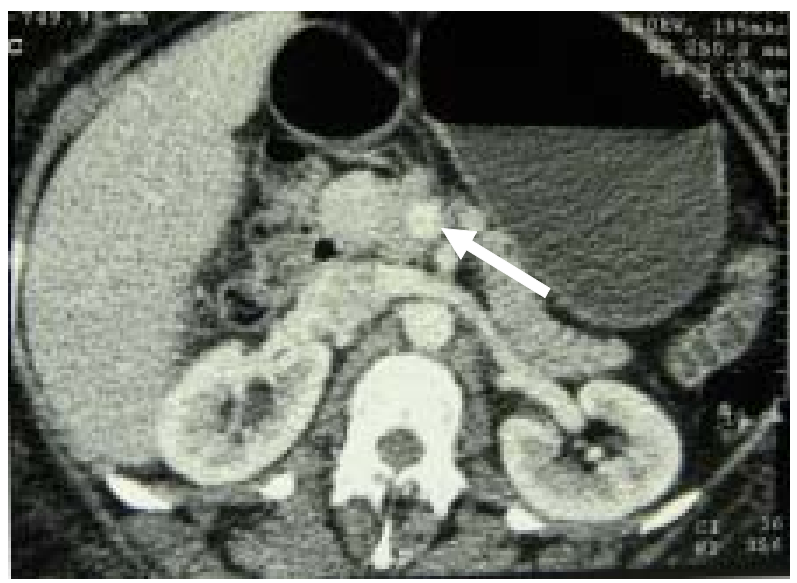

Figure 1. Triple phase CT scan of the abdomen in early arterial phase. White arrow shows the enhanced tumour in the head of the pancreas.

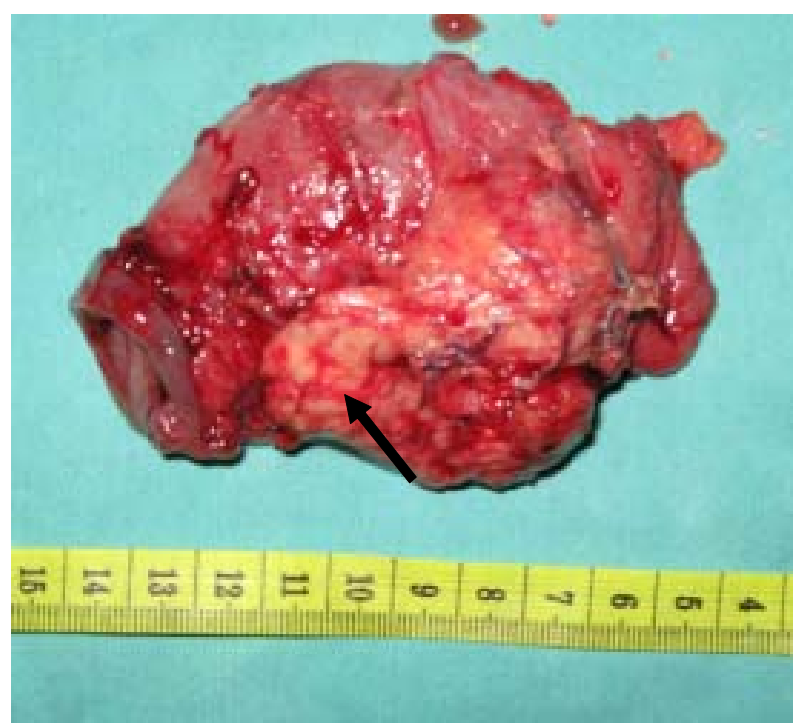

Figure 2. Black arrow showing the specimen of the tumour removed during surgery.

Histopathological examination of the pancreatic mass confirmed a benign insulinoma of the pancreas. The patient was discharged home and is being followed up as an outpatient for the control of her post-pancreatectomy diabetes mellitus. Antiepileptic drugs have been discontinued with no recurrence of seizures.

\section{Discussion}

Diagnosis of insulinoma may be delayed if symptoms are nonspecific. The common clinical manifestation of an insulinoma is fasting hypoglycaemia, with discrete episodes of neuroglycopenic symptoms that may or may not be preceded by sympathoadrenal (autonomic) symptoms. Neuroglycopenic symptoms are predominant in some patients and these patients may be diagnosed as epilepsy or neuropsychiatric disease $(3,4)$. In a retrospective study of 59 patients with histologically confirmed islet cell adenomas, the interval between onset of symptoms and diagnosis ranged from 1 month to 30 years, with a median of 24 months. A significant proportion $(39 \%)$ was originally diagnosed with a seizure disorder $(6,7)$.

The biochemical hallmark of diagnosis of insulinoma is the fasting hypoglycaemia in the face of inappropriately raised serum insulin and $\mathrm{C}$-peptide levels and exclusion of surreptitious intake of exogenous insulin or oral hypoglycaemic agents (5).

Successful preoperative tumour localisation is achieved in about $60 \%$ of patients.

The imaging modalities used for localisation of insulinoma are:

- Transabdominal ultrasound: readily available, inexpensive, and noninvasive with detection rate of $25-65 \%$.

- Endoscopic ultrasound: equipment and expertise are not widely available but with detection rate of $70 \%$.

- CT: widely available and non-invasive with a detection rate of $70 \%$ when contrast is used.

- MRI, selective angiography, percutanous transhepatic portal venous sampling, arterial simulation venous sampling and radionuclide imaging can also be used with sensitivities ranging from 50 to $90 \%(4,5,9-11)$.

In addition to the preoperative diagnostic imaging techniques, intraoperative palpation and ultrasound are the gold standards for localising an insulinoma, with a reported success rate of $96-100 \%(9,12)$. Surgery is the only curative treatment of this potentially lethal condition. The nature of surgery depends upon the size, location and resectability of insulinoma. Simple enucleation is the most favoured option for solitary insulinoma, while distal pancreatectomy with or without splenectomy is preferred for multiple insulinomas occurring in the body and tail of the pancreas. Pancreatoduodenectomy (Whipple's procedure) becomes indispensable for a non-enucleable insulinoma in the head and neck regions of the pancreas $(9,10)$. The surgical procedure is usually chosen by the surgeon, and the incidence of post surgical complications is approximately $10 \%(8,9,13)$.

Medical treatment is continued when surgery is contraindicated. Streptozocin, diazoxide, verapamil, 
pheytoin, somatostatin can be used for the medical treatment $(11,12)$.

Malignant insulinomas are rare, and therefore, little is known about their clinical presentation and long term prognosis. They are generally indolent tumours, and long survival has been reported, even in the presence of liver or lymph node metastases $(12,15)$.

To conclude, insulinoma is a rare disease even at large centres. With its vague and varied clinical presentation, insulinoma can be a diagnostic conundrum. Diagnosis of insulinoma should be considered in any patient with unusual neurological features, including atypical convulsions refractory to pharmacotherapy.

\section{References}

1. Poornima V, Mahale A, Kumar A, Chandra S, Paudel K. Insulinoma - a case report and review of diagnostic modalities. JIACM 2008; 9(1): 53-6.

2. Modlin IM, Tang LH. Approaches to the diagnosis of gut neuroendocrine tumors: the last word (today). Gastroenterology 1997; 112(2): 583 - 90.

3. Rehman A. Insulinoma - a deceptive endocrine tumour. $J$ Pak Med Assoc 2011; 61(9): 911 - 4.

4. Ozkaya M, Yuzbasioglu MF, Koruk I, Cakal E, Sahin M, Cakal B. Preoperative detection of insulinomas: two case reports. Cases Journal 2008; 1: 362.

5. Boukhman MP, Karam JM, Shaver J, et al. Localization of insulinomas. Arch Surg 1999; 134(8): 818 -22; discussion $822-3$.

6. Nikafarjam M, Warshaw A, Axelrod L, Deshpande V, Thayer $\mathrm{S}$, Ferrone $\mathrm{C}$, et al. Improved contemporary surgical management of insulinoma: a 25-year experience at the Massachusettes General Hospital. Ann Surg 2008; 247: $165-72$.

7. Liu H, Peng C, Zhang S, Wu Y, Fang H, Sheng H, et al. Strategy for surgical management of insulinomas: analysis of 52 cases. Dig Surg 2007; 24: 463 - 70.

8. Mansour JC, Chen H. Pancreatic endocrine tumour. Journal of Surgical Research 2003; 120: 139-61.

9. Abboud B, Boujaoude J. Occult sporadic insulinoma: localization and surgical strategy. World J Gastroenterology 2008; 14(5): $657-65$.

10. Mouaqit $\mathrm{O}$, Boubouh A, Ifrine L, Malki EHOE, Mohsine $\mathrm{R}$, Belkouchi A. Insulinoma misdiagnosed as epilepsy. Open Journal Of Gastroenterology 2012; 2: 28 -30.

11. Mittendorf EA, Liu YC, McHenry CR. Giant insulinoma: case report and review of the literature. The Journal of Clinical Endocrinology and Metabolism 2005; 90 (1): 575 80 .

12. Rassouli A, Lai JH, Sargeant HR. Insulinoma - an atypical presentation: case report and literature review. University of Toronto Medical Journal 2004; 82(1): 36 - 41.

13. Graves TD, Gandhi S, Smith SJM, Sisodiya SM, Conway GS. Misdiagnosis of seizures: insulinoma presenting as adult-onset seizure disorder. J Neurol Neurosurg Psychiatry 2004; 75: 1091 - 2 .

14. Suzuki K, Miyamoto M, Miyamoto T, Hirata K. Insulinoma with early-morning abnormal behaviour. Intern Med 2007; 46(7): $405-8$.

15. Tran TH, Pathak RD, Basa ALP. Metastatic insulinoma: case report and review of the literature. South Med J 2004; 97(2): $199-201$. 